\title{
Variación sociolingüística en el empleo de un nuevo marcador discursivo: ahora sí que en el español de México
}

\author{
Juan Diego Aldama Peñaloza ${ }^{1}$ \\ Asela Reig Alamillo \\ Universidad Autónoma Metropolitana, México
}

\begin{abstract}
Resumen
En este artículo se analiza la distribución del marcador discursivo ahora sí (que) en dos variedades del español de México, Ciudad de México y Monterrey, y en dos momentos diferentes. Empleando los corpora de PRESEEA de ambas ciudades y otros dos corpora de entrevistas sociolingüísticas más antiguos, el estudio constata diferencias diacrónicas y diatópicas claras: el marcador es más frecuente en Ciudad de México que en Monterrey y, en ambas variedades, es más frecuente en los datos más recientes. Además, el análisis de frecuencias del marcador apunta interesantes diferencias sociales en el empleo de ahora sí (que) en la Ciudad de México, donde su uso está favorecido por hablantes jóvenes, por hombres y por informantes de los niveles de instrucción bajo y medio.
\end{abstract}

Palabras clave: variación pragmática, variación discursiva, ahora sí que, marcadores del discurso México.

1 Para correspondencia, dirigirse a: Juan Diego Aldama Peñaloza (didacus714@gmail. com) o a Asela Reig (asselareig@gmail.com), Departamento de Filosofía, Universidad Autónoma Metropolitana. San Rafael Atlixco 186, Iztapalapa, Vicentina, 09340 Ciudad de México, D.F., México. 


\title{
SOCIOLINGUISTIC VARIATION IN THE USE OF A NEW DISCOURSE MARKER: AHORA SÍ QUE IN MEXICAN SPANISH
}

\begin{abstract}
This article analyzes the distribution of the discourse marker ahora si (que) in two varieties of Mexican Spanish, Mexico City and Monterrey, and at two different time periods. Making use of the PRESEEA corpora from both cities and another two corpora of sociolinguistic interviews collected several decades ago, the study finds clear diachronic and geographic differences: the discourse marker is more frequent in Mexico City than in Monterrey and, in both varieties, it is more frequent in the more recent corpora. Moreover, the frequency analysis of the discourse marker shows interesting social differences in the use of ahora si (que) in Mexico City, where it is favored by young speakers, men and speakers of low and medium instruction.
\end{abstract}

Keywords: pragmatics variation, discourse variation, ahora sí que, discourse markers in Mexican Spanish.

Recibido: 23/02/2016 Aceptado: 04/07/2016

\section{INTRODUCCIÓN}

El presente trabajo analiza un fenómeno de variación en el nivel discursivo, el empleo del marcador ahora sí que, y sus formas reducidas ahora sí y ora sí (que), en español de México (1), empleando dos corpus pertenecientes al proyecto PRESEEA: los de la Ciudad de México y Monterrey, México.

(1) I: convivimos como familia porque es el día en que / verdaderamente nos juntamos todos los que / somos hijos ahora sí que / del mismo padre / la misma madre // o sea como familia (CM)

A pesar de la muy prolífica producción de trabajos sobre marcadores discursivos en español (Portolés 1998; Martín Zorraquino y Montolío 1998; Martín Zorraquino y Portolés 1998; Santos 2003), estos están, en su mayor parte, centrados en usos del español de la Península ibérica o en usos que se consideran panhispánicos. Aunque los trabajos sobre marcadores discursivos 
en otras variedades del español han empezado a aportar datos sobre diferentes marcadores o diferentes valores de los señalados antes, aún queda mucho por hacer para tener un panorama más completo de los marcadores discursivos existentes en español, su variedad dialectal y, dentro de cada variedad, su relación con la variación sociolingüística.

Es en este ámbito en el que se sitúa el presente trabajo, que consiste en una primera aproximación al marcador discursivo del español de México ahora sí (que), que, según nuestro conocimiento, no ha sido señalado ni analizado hasta la fecha. La percepción de partida es que este marcador es reciente y observar su distribución sociodemográfica en datos de habla real permitirá concretar su expansión y frecuencia entre los hablantes de las dos ciudades de México mencionadas.

Este trabajo analiza la aparición de ahora sí (que) en dos corpus de PRESEEA, Ciudad de México y Monterrey, México, y contrasta estos datos con los procedentes de corpora más antiguos de entrevistas sociolingüísticas recogidas en las mismas ciudades. Con este análisis se ilustra que el material recogido en el Proyecto de PRESEEA aporta interesantes datos no solo para el estudio de la variación fonética y morfosintáctica, sino también para la variación pragmático-discursiva. A pesar de las características y complicaciones que este nivel de análisis presenta todavía, y que se comentarán más adelante, contar con corpora comparables de diferentes variedades del español y con información sociodemográfica de sus hablantes permite explorar la interesante relación entre el empleo de partículas discursivas y la estratificación social de los hablantes y comenzar a ampliar nuestro conocimiento de variación geográfica en el empleo y valores de los marcadores discursivos del español.

\section{VARIACIÓN Y MARCADORES DISCURSIVOS}

Tras el bien conocido debate sobre la (in)conveniencia de extender el marco de estudio variacionista y su metodología más allá del nivel fonológico a fenómenos morfosintácticos, inaugurado con el ya clásico artículo de Lavandera (1978), nos encontramos, desde hace pocos años, ante el siguiente paso: la extensión, aún más allá, de los límites de la metodología variacionista al análisis de fenómenos discursivos. Si la primera de estas extensiones supuso aceptar nuevas características (Silva-Corvalán 2001:130; Schwenter 2011), el ajuste del método variacionista al plano pragmático- 
discursivo no se encuentra exento de discusión tanto en el aspecto teórico como en el metodológico. Abordar el estudio de marcadores discursivos con una visión variacionista exige enfrentar, de entrada, las siguientes cuestiones (Pichler 2010). En primer lugar, su frecuencia es, en general, baja, lo que constituye un reto para los estudios cuantitativos. Además, en lo que respecta a la conveniencia de aplicar el concepto de variable a estas unidades discursivas, Pichler señala que su multifuncionalidad o polifuncionalidad impide que las variantes discursivas sean identificadas con base en criterios de equivalencia semántica y que esa misma multifuncionalidad genera dificultades para delimitar su contexto variable. La reflexión y discusión sobre cómo acomodar, si es que debe hacerse, el concepto de variable al estudio cuantitativo de estos elementos discursivos está sobre la mesa, así como otros asuntos relativos al rigor metodológico de estos estudios (Cortés 1998; San Martín 2004-2005, 2013; Carbonero y Santana 2010; Andersen 2001).

A pesar de que el estudio variacionista de unidades discursivas y pragmáticas se encuentra en un estado embrionario, en gran medida debido a la falta de un consenso bien establecido en los planos teóricos y metodológicos, desde hace años se antoja necesario hacer confluir los intereses de la pragmática y la sociolingüística y observar la distribución sociodemográfica y estilística de marcadores discursivos (y otras unidades de análisis pragmático-discursivo) y se va consolidando el reconocimiento de que unidades como eh, pues, bueno, o sea, etc., poseen, además de uno o varios significados o funciones lingüístico-discursivas, significado social y que, al igual que otras unidades de la gramática, su uso es heterogéneamente ordenado y su frecuencia de uso se correlaciona con factores sociales, estilísticos y también internos de la lengua. Este enfoque de estudio resulta, sin duda, promisoria, en palabras de San Martín (2004-2005: 213).

Dentro de la muy numerosa bibliografía relativa a marcadores discursivos que se ha generado en las últimas décadas, la cantidad de trabajos que abordan su uso teniendo en cuenta factores extralingüísticos ha sido relativamente limitada y es aún heterogénea en su metodología y conclusiones.

Carbonero y Santana (2010) recogen investigaciones que reconocen, implícita o explícitamente, la pertinencia de abordar la variación en marcadores discursivos y que examinan uno o varios marcadores prestando atención a las características sociales de los hablantes, en trabajos con datos de diferentes zonas de España y de Latinoamérica. Los trabajos que se reseñan se dividen entre los que estudian marcadores en alguna zona geográfica en España y aquellos que observan alguna variedad americana. Entre los primeros, se encuentran trabajos de Cortés (1991) en el habla de León, o Fuentes (1990a y b; 1993a y b) en el habla urbana de Sevilla, 
quienes observan la distribución por grupos sociales de un buen número de marcadores propios de la expresión oral. Garcés (1994a y b) analiza entonces y pues en Málaga, y Serrano $(1995,2001)$ analiza la verdad y pues. En Hispanoamérica, Rodríguez Cadena (1999) analiza marcadores en Barranquilla, Colombia; centrándose en discurso emitido por mujeres, Cepeda y Poblete (1999) analizan su uso de marcadores discursivos en el habla de Valdivia, Chile, y Álvarez y Joven (2005) estudian marcadores en el habla de Mérida, Venezuela. En estos trabajos, en general, el objetivo principal no era, sin embargo, atender a la posible función del o los marcadores como muestras de estratificación social y, en general, no se entra en la discusión teórica o metodológica de la variación discursiva en relación con otros niveles de variación.

A los trabajos reseñados en Carbonero y Santana (2010) pueden añadirse algunos otros. Cestero y Moreno (2008) analizan los marcadores jvenga! y vale en el barrio de Salamanca en Madrid. Además del análisis cualitativo, que incluía el examen de sus funciones (entre ellas, aceptación, reproche con ironía, asentimiento, consentimiento por obligación o reservas) y características formales, se realiza un análisis cuantitativo que incorpora la frecuencia de uso de sus valores y su uso en relación con variables lingüísticas contextuales y sociales, considerando sexo, edad, nivel de estudios, clase social y de contexto lingüístico en que aparecen los marcadores (tipo de enunciado y de discurso). Su estudio ilustra una de las dificultades que este tipo de investigaciones encuentra, como se señalaba arriba: la baja frecuencia de estas construcciones, que dificulta un análisis cuantitativo. Del análisis de 36 entrevistas, se obtienen 42 ocurrencias de vale, y de 26 entrevistas analizadas, 36 casos de jvenga! Del uso escaso en la entrevista semidirigida, los autores concluyen que ambos marcadores podrían estar en un proceso de generalización y difusión desde el registro más informal, de manera que su uso parece indicar un caso de variación diafásica. Asimismo, a partir de análisis cuantitativo de sus datos señalan que los marcadores son "sociopragmáticos", pues son usados más por mujeres que por hombres y los jóvenes y los adultos los usan más que los mayores, lo que se interpreta como una progresiva extensión de su uso.

Orozco (2014) analiza los marcadores discursivos ¿no?, eh y ¿verdad? en cuanto a su distribución a lo largo de una situación de entrevista sociolingüística, los contextos en los que aparece, su frecuencia de aparición, su función pragmática (expresivo-fática o apelativa) y su uso por hablante. Para el análisis cuantitativo se tomaron en cuenta los factores sociales edad, sexo y el tipo de diada entre los interlocutores (hombre-hombre, mujer-mujer, mujer-hombre) y solo se incluyó un grupo social (alto). Con estos parámetros de análisis, la autora concluye que un factor que propicia la aparición de estos 
marcadores en este tipo de intercambio es la necesidad de los participantes de involucrar al interlocutor, así como de corroborar su postura ante lo dicho. El análisis cuantitativo, en cambio, no mostró ningún patrón reseñable en relación con el género y los papeles sociales de los hablantes.

San Martín (2004-2005) analiza la función pragmática y la estratificación sociolingüística de igual como reformulador de distanciamiento. En el análisis de 72 entrevistas sociolingüísticas (533 casos de igual) encuentra que esta función de igual se presenta más frecuentemente en el habla de los jóvenes de nivel sociocultural medio, especialmente mujeres. En San Martín (2013), el autor analiza el uso de los reformuladores de distanciamiento en la mitad del corpus de PRESEEA Chile y encuentra, de nuevo, que el reformulador igual, mucho más frecuente que los demás reformuladores de distanciamiento, está muy extendido entre los hablantes jóvenes de la muestra (entre 20 y 34 años) y apunta que podría tratarse de un fenómeno de expansión del marcador en esta variedad del español.

Diferente objetivo dentro de la observación de la variación en marcadores es el de Santana (2014), quien aborda la posible diferencia diacrónica en el empleo de marcadores discursivos, en general, en los hablantes cultos de Sevilla. La autora compara las frecuencias generales de los marcadores en 6 entrevistas sociolingüísticas de cada época (1972-73 y 2012-13) y reporta que no se aprecian diferencias significativas en el tipo de marcadores empleados, ni tampoco diferencias en la frecuencia de marcadores (en general) por sexo o edad.

Los trabajos señalados coinciden, por tanto, en un interés general sobre la información que corpora sociolingüísticos existentes pueden ya ofrecer sobre la distribución diatópica, diafásica, distrática o diacrónica de marcadores discursivos. La selección de los marcadores que se analizan no siempre se justifica, o no siempre de igual manera (marcadores que cumplen una misma función discursiva, marcadores en general, etc.); el análisis cuantitativo no siempre asegura la comparabilidad de los datos incluyendo algún tipo de cálculo de proporción con la longitud de la entrevista; y la cantidad de datos encontrados y analizados varía enormemente de estudio a estudio, por mencionar algunas de las diferencias. Estos estudios, a pesar de la intuición general que se indicaba arriba, no muestran, de forma consistente, que los marcadores analizados estén en clara correlación con datos sociodemográficos de los hablantes, pero las características de las muestras y de los propios análisis hacen estos resultados, de momento, poco concluyentes.

En inglés, por el contrario, son varios los trabajos que sí encuentran correlaciones bastante claras entre el empleo de determinadas construcciones de uso discursivo y las características sociodemográficas de sus hablantes. Tagliamonte (2005) analiza just, like e intensificadores como so y really, 
unidades muy asociadas al habla de tres grupos etarios: preadolescentes, adolescentes y jóvenes. El análisis incluía factores externos (edad, sexo, clase social) y la posición de like y de just con respecto a la unidad lingüística que le sigue (verbos, nombres y oraciones) y encuentra que la distribución de estos elementos tiene que ver con la edad (de diferentes modos: su uso durante un solo periodo de vida en el caso de like; aumento de frecuencia con el aumento de edad en el caso de just y de so, en este último solo en la preadolescencia y adolescencia) y con el sexo del hablante (mujeres más frecuencia que hombres en like y en just y en so).

Andersen (2001) analiza el marcador pragmático like y las preguntas finales de enunciados (invariant tags e invariant follow-ups, como is it? $e$ innit?) en inglés en el habla de adolescentes londinenses, analizando sus funciones pragmáticas y la variación de esos fenómenos en el corpus analizado. Su análisis observa los rasgos de los hablantes y concluye que tanto is it? como innit? están generalizados aunque la segunda forma es menos frecuente en hablantes blancos de clase alta y parece estarse extendiendo a partir del habla de los varones. Respecto a like, este es principalmente usado por mujeres blancas en la adolescencia tardía y es ligeramente más frecuente en la clase social alta que en las demás.

La expresión you know ha sido también estudiada con este enfoque (Erman 2001) y se asocia en general con el habla de adolescentes. Pichler (2013) analiza las formas I don't know y I don't think, y las variantes formales de cada expresión (I dono, I duno) en el habla de 36 informantes del norte de Inglaterra. Además de mostrar que las funciones discursivas condicionan la aparición de las variantes más que los factores sociales, encuentra que algunas variantes (I divn't knaa, I divn't think) son afectadas por factores sociales.

La categoría de los llamados en inglés general extenders (and stuff like that, en inglés) es otro de los fenómenos discursivos bastante estudiados desde esta perspectiva: su empleo parece ser más frecuente en el habla de los jóvenes, y en varios estudios se encuentra correlación con el sexo y la clase social (Dubois 1992; Cheshire 2007; Pichler y Levey 2011).

En resumen, esta breve revisión de algunos trabajos dedicados a la variación en marcadores discursivos pretende ilustrar que, en la actualidad, esta es un área de estudio incipiente pero prometedora, de la que es esperable que encontremos, en los próximos años, interesantes aportaciones que nos permitan profundizar en nuestro conocimiento de la variación pragmática. Schneider (2010) señala que la pragmática variacionista - que incluye, junto al estudio de marcadores discursivos en el sentido amplio, otros objetos de estudio como actos de habla o secuencias interaccionales- puede definirse como "el estudio de la variación intralingüística y macro social" (Schneider 2010: 250) 
en el nivel pragmático. Con esta definición el autor quiere llamar la atención sobre la necesidad de ampliar los estudios contrastivos entre variedades de una misma lengua (frente a trabajos, en su opinión más frecuentes, que se enfocan en diferencias pragmáticas entre lenguas) y profundizar en la relación entre los fenómenos pragmático-discursivos y los rasgos socio-demográficos como edad, género, etnicidad, clase social y región.

Es a esta parcela del estudio lingüístico a la que contribuye el presente estudio del marcador ahora sí (que) en dos variedades del español de México, haciendo uso de los corpus de PRESEEA ya accesibles para los investigadores.

\section{AHORA SÍ QUE COMO MARCADOR DISCURSIVO}

Aunque no existe una definición única del concepto de marcador, se aceptan en general los siguientes puntos para su caracterización:

a) No constituyen una clase uniforme de palabras, en el sentido de que no es posible establecer una relación biunívoca entre una categoría gramatical dada y su funcionamiento como marcador discursivo.

b) Son unidades lingüísticas (más o menos) invariables, fijadas en su forma.

c) No contribuyen a las condiciones de verdad del enunciado, sino que aportan otro tipo de significado al enunciado. Este significado es, según algunos autores (Portolés 1998), un significado de procesamiento, es decir, guían las inferencias que deben obtenerse del enunciado en el que se insertan.

d) Tampoco contribuyen a las relaciones sintácticas de la oración.

e) El surgimiento de las funciones pragmáticas que desempeñan coincide con la desemantización del significado conceptual de la expresión en origen.

f) Con diferencias de marcador a marcador, suelen tener cierta libertad de posición en relación con el fragmento discursivo al que acompañan y, aunque no siempre es así, con frecuencia constituyen incisos entonativos, delimitados por pausas.

g) Los elementos discursivos que analizamos pueden parecer, además, con miembros discursivos que se corresponden con diversas categorías léxicas y sintagmáticas, no solo con oraciones, y algunos pueden constituir enunciados por sí mismos. 
El significado de ahora sí (que) queda fuera de los límites de este artículo pero resulta necesario caracterizar, al menos someramente, ahora sí (que) como un marcador discursivo para explicar qué usos de esta construcción se incluyen en el análisis que se presentará en los apartados posteriores.

La construcción ahora sí (que) se emplea en español de México tanto con su valor léxico original (valor temporal y de contraste) como con valor discursivo. En el primer caso, ilustrado en $(2 a)^{2}$, lleva a cabo un papel sintáctico en la oración y mantiene su significado de expresión temporal (ahora) y el valor contrastivo del adverbio afirmativo sí. En cambio, en el segundo, ilustrado en (2b), ahora sí que ha perdido el valor semántico original a favor de un valor pragmático y queda fuera de la estructura sintáctica del enunciado en que aparece.

(2a) E: ¿por qué?/ ¿qué pasó?

I: mira// yo tenía unos inmensos deseos

EF: $m h$

I: de estudiar/ pero inmensos// y hago mi examen para la preparatoria/soy la primera que salgo/ me siento muy segura de mí misma/ dije/ "yo me saco mínimo un nueve"

$\mathrm{E}: \mathrm{mh}$

I: sí/ pues $<\sim$ pus $>$ me regresan mis papeles y me dicen que/ "ahí < ai $>$ para la próxima/ pero tengo una calificación/ de tres punto cinco"/ yo dije/ "no/ estos están locos// ¿cómo crees?/ tres punto cinco/ ¡en mi vida! yo he sacado una calificación de esas"// lloré hasta que me cansé/ y al siguiente año dije/ "ya me preparé/ ya estudié más/ y ahora sí la voy a hacer"// hago mi examen para la prepa/me vuelven a regresar mis papales/ con cinco punto tres/ nomás me la cambiaron (risa) (CSCM)

(2b) entonces $<\sim$ tos $>/$ pues $<\sim$ pus $>$ yo cada que tengo/ que estoy dando rondines/ tengo que checar/ ahora $<\sim$ ora $>$ sí que los locales que estén ahí/ ya sean/ vinaterías// hay cantinas (CSCM, Entrevista 41).

Prueba de que el primer ejemplo mantiene un valor temporal adverbial es que puede ser parafraseado con esta vez sí que, mientras que el segundo no:

\footnotetext{
2 Tras cada ejemplo se indica el corpus de procedencia con iniciales: Corpus Sociolingüístico de la Ciudad de México (CSCM), Corpus de Monterrey (CM) y Corpus del Habla de la ciudad de México (CHCM).
} 
(3a) y al siguiente año dije/ "ya me preparé/ ya estudié más/ y esta vez si la voy a hacer"//

(3b) ?? entonces $<\sim$ tos $>/$ pues $<\sim$ pus $>$ yo cada que tengo/ que estoy dando rondines/ tengo que checar/ esta vez sí que los locales que estén ahí/ ya sean/ vinaterías// hay cantinas.

Tanto en (2a) como en (3a), ahora sí que y esta vez sí que aportan un valor temporal (ahora / esta vez) y un contraste con una ocasión anterior, que está presupuesta en la expresión literal ahora sí que (y, de igual manera, en esta vez sí que). En cambio, en (3b), la expresión ahora sí que no está dando cuenta de ninguna relación temporal con respecto a la perífrasis verbal tengo que checar, por lo que se puede afirmar que, a pesar de estar constituida por dos adverbios, ninguno de ellos retiene -al menos del todosu significado léxico. Estamos ante el marcador discursivo. Por la misma razón, el significado lógico-referencial de los enunciados donde aparece ahora sí (que) en los ejemplos de (2) solo se ve afectado si se suprime la frase en (2a), mientras que en (2b) no ocurre lo mismo:

(4a) y al siguiente año dije/ "ya me preparé/ ya estudié más/ y la voy a hacer".

(4b) entonces $<\sim$ tos $>$ / pues $<\sim$ pus $>$ yo cada que tengo/ que estoy dando rondines/ tengo que checar/ ø los locales que estén ahí/ ya sean/ vinaterías// hay cantinas

El significado lógico-referencial de (4a) pierde información respecto al de (2a), mientras que eso no ocurre en (4b) respecto a (2b).

Ahora sí (que) parece no poder ser colocado como inciso con respecto a su entonación y, por otro lado, no puede aparecer en una posición diferente a la de inicio del constituyente sobre el que incide:

(5a) ?? sí/ yo hablaba seguido con ella/ procuraba precisamente no perder comunicación/ para// ipues < pus > para que me tuvieran presentes!/ por equis/ o por ye/ ahora $<\sim$ ora $>$ si que uno nu- nunca sabe/ uno pone/ Dios dispone/ no sabes// tú dices/"voy y vengo"/ pero/ o sea sales/ pero sabes si regresas/ [cada] (CSCM, Entrevista 52)

(5b) ipues $<\sim$ pus $>$ para que me tuvieran presentes!/ por equis/ o por ye/ ahora $<\sim$ ora $>$ sí que, uno nu- nunca sabe/

La coma -colocada por nosotros en (5b) y ausente en (5a)- que aparece después del marcador da cuenta de su dudoso estatus como inciso. Asimismo, no es posible encontrarlo en alguna otra posición que no sea la inicial con 
respecto a la unidad lingüística sobre la que incide. Así, parece adherirse al grupo de marcadores que se usan en posición inicial con respecto al constituyente sobre el que incide, como de ahi y es decir (Zorraquino y Portolés 1999: 4063). Este hecho sobre la posición del marcador también es atribuible al marcador ahora sí, sin embargo, su estatus como inciso no parece ser dudoso:

(6) mi hermano y yo pues $<\sim$ pus $>$ estamos en el plan de/ echarle la mano/ ayudarle/ $\mathrm{y}<\sim \mathrm{y}:>/$ aprender igual/ para posteriormente tratar de hacer lo mismo/ no sabemos ahora < ora $>$ sil a ciencia cierta si/ si nos váyamos (sic) a dedicar de lleno con este mismo trabajo o $<\sim 0$ : $>/$ o pueda cambiar/ (CSCM, Entrevista 52)

Como queda indicado por la diagonal -que es parte de la transcripción e indica la separación de segmentos prosódicos diferentes-, el marcador ahora $s i$ indica que prosódicamente está separado del fragmento discursivo sobre el que incide -en este caso, un sintagma prepositivo.

Aunque, como se ha dicho ya, el estudio detallado del significado de ahora sí (que) no forma parte de los objetivos de este artículo, apuntaremos, aún a riesgo de ofrecer una caracterización muy preliminar, que ahora sí (que) parece ser un marcador discursivo con instrucciones de formulación (Portolés 1998: 103). Este grupo de marcadores presentan el miembro discursivo que introducen el miembro discursivo en el que aparecen como aquel que transmite satisfactoriamente la intención comunicativa del hablante (Portolés 1998, Murillo 2000).

Encontramos empleos de ahora sí que en los que parece evidente su función como reformulador, es decir, un marcador que permite al hablante ofrecer una nueva formulación de aquello que acaba de decirse, para corregirlo, explicarlo, modificar sus elecciones léxicas, etc. En el ejemplo (7), en el que se habla de las condiciones dentro de la cárcel y cómo allí se realizan "negocios", el hablante se autointerrumpe (todos los patrulle-/ todo) y reinicia reformulando. Esa reformulación (todo todo se mueve) está introducida con ahora sí que.

(7) E: [pero] pues sí/ ellos están fomentando eso ¿no? pues < pus> también como [que]

I: [ajá]/ pero es que todo es negocio

E: ajá

I: todos los patrulle-/ todo ahora $<\sim$ ora $>$ sí que todo todo se mueve ahí adentro// ¿cómo ves? (CSCM, Entrevista 74) 
No obstante, no todos los empleos de ahora sí (que) son reformuladores, es decir, no en todos los casos aparece un primer miembro discursivo que es modificado o reformulado. En muchas ocasiones, no se presenta una nueva formulación de un miembro precedente sino que, parece, ahora sí (que) solo indica aquello que introduce como el miembro del discurso que ha de tenerse en cuenta para la prosecución del discurso (8) (Portolés 1998: 105).

(8) E: pero si la muchacha se quiso ir ¿no?/ o ¿qué?/ o

I: pues sí haz de cuenta que la primera audi-/ la primera audiencia/ la < la:> la muchacha lo defendió/ pero ya en la segunda ya no

E: ya no/ ella también/ [ella se]

I: [ajá]

E: volteó más bien

I: mm pues $<\sim$ pus $>$ la volteó su papá/ ahora $<\sim$ ora $>$ sí que con dinero

E: mm (CSCM. Entrevista 74)

No trataremos de ofrecer aquí un análisis detallado del valor de ahora sí (que) pero, al menos en los datos analizados, la gramaticalización de esta expresión con valor discursivo no ha desarrollado un valor de oposición, como el que el marcador discursivo ahora, que evidentemente comparte con ahora sí (que) el adverbio ahora en la expresión de origen, parece presentar en la mayoría de sus empleos (Silva Corvalán 2001: 88\%).

En resumen, el valor de ahora sí (que) que se incluye en este análisis es el del marcador discursivo, con instrucciones de formulación, que coexiste con la expresión original en la que los adverbios mantienen su significado, por lo que la expresión original aporta un valor adverbial temporal y la presuposición de un evento anterior -temporalmente- con el que hay un contraste. Retomaremos esto en el siguiente apartado.

\section{METODOLOGÍA}

\subsection{CORPus}

Los datos analizados en este estudio proceden de cuatro corpora, que representan el habla de dos ciudades de México en dos momentos diferentes. Los dos más recientes son el Corpus Sociolingüístico de la 
Ciudad de México (Martín Butragueño y Lastra 2011, 2012) y el Corpus El Habla de Monterrey - PRESEEA (Rodríguez Alfano, Flores Treviño y Pérez Aguirre 2012), ambos pertenecientes al Proyecto para el Estudio Sociolingüístico del español de España y de América (PRESEEA). En ellos se incluyen las variables sexo (hombre, mujer), edad (20-34 años; 35-54 años; 55 o más) y nivel de instrucción (nivel bajo: analfabetos, sin estudios o con enseñanza primaria. Alrededor de cinco años de escolarización; nivel medio: enseñanza secundaria. De diez a doce años de escolarización; nivel alto: enseñanza superior -universidad, técnica superior-. Alrededor de 15 años de escolarización).

Cada uno de ellos está compuesto por 108 entrevistas, con muestreo intencionado. La Tabla 1 muestra la distribución de entrevistas por casilla para cada uno de los corpora de PRESEEA que aquí se analizan.

Tabla 1. Número total de entrevistas por casilla en el CSCM y el CM-PRESEEA

\begin{tabular}{|l|c|c|c|c|c|c|}
\hline & \multicolumn{2}{|c|}{ Escolaridad baja } & \multicolumn{2}{c|}{ Escolaridad media } & \multicolumn{2}{c|}{ Escolaridad alta } \\
\hline Edad & H & M & H & M & H & M \\
\hline Jóvenes & 6 & 6 & 6 & 6 & 6 & 6 \\
\hline Adultos & 6 & 6 & 6 & 6 & 6 & 6 \\
\hline Mayores & 6 & 6 & 6 & 6 & 6 & 6 \\
\hline
\end{tabular}

Los otros dos corpora incluidos en el estudio para comparar diacrónicamente la frecuencia de uso del marcador son el Corpus del Habla de Monterrey (CHM) (Rodríguez Alfano 2010), creado en los años ochenta, y el corpus El habla de la Ciudad de México (CHCM) (Lope Blanch 1971, 1976). La Tabla 2 ofrece la distribución por factores sociales de la muestra de 154 entrevistas del Corpus del Habla de Monterrey considerada para este trabajo:

Tabla 2. Número de entrevistas del CHM consultadas para este trabajo

\begin{tabular}{|l|c|c|c|c|c|c|}
\hline & \multicolumn{2}{|c|}{ Escolaridad baja } & \multicolumn{2}{c|}{ Escolaridad media } & \multicolumn{2}{c|}{ Escolaridad alta } \\
\hline Edad & H & M & H & M & H & M \\
\hline Jóvenes & 9 & 12 & 9 & 8 & 15 & 12 \\
\hline Adultos & 8 & 13 & 9 & 9 & 12 & 10 \\
\hline Mayores & 14 & 9 & 3 & 0 & 1 & 1 \\
\hline
\end{tabular}

En este corpus, los hablantes más representados son los informantes jóvenes. Se incluyeron en el análisis los pocos hablantes mayores de nivel bajo y medio que fueron entrevistados. 
El Habla de la Ciudad de México, recogido entre 1967 y 1974, incluye las muestras del habla popular, entrevistas hechas a informantes con un nivel elemental de estudios y que ejercen ocupaciones no profesionales, y las muestras de la norma culta, entrevistas realizadas a personas con un nivel de estudios de licenciatura o mayor y que ejercen una actividad laboral relacionada con su preparación académica. Los factores sexo y edad no cuentan con un número equilibrado de informantes. Este corpus tiene un total de 83 informantes -42 en el Habla Popular y 41 en la Norma Culta- en un total de 66 entrevistas repartidas en 34 y 32 en cada muestra, respectivamente. Nótese la ausencia de un estrato que equivalga al nivel medio de instrucción presente en los dos corpus anteriores. En cada trascripción, se dan datos de los informantes como sexo, edad, lugar de procedencia, nivel cursado de estudios, ocupación y procedencia de los padres. A las entrevistas incluidas en los tomos editados originalmente por Lope Blanch se suman las nuevas transcripciones de la Norma Culta. Esta nueva parte del corpus se compone de 24 entrevistas realizadas a 46 hablantes de nivel alto de instrucción (Jóvenes: 13 hombres, 14 mujeres; Adultos: 4 hombres, 9 mujeres y Mayores: 2 hombres, 4 mujeres). La Tabla 3 muestra la distribución de las entrevistas del CHCM:

Tabla 3. Número total de entrevistas por casilla en el CHCM (con transcripciones nuevas)

\begin{tabular}{|l|c|c|c|c|}
\hline & \multicolumn{2}{|c|}{ Habla popular } & \multicolumn{2}{c|}{ Norma culta } \\
\hline Edad & H & M & H & M \\
\hline Jóvenes & 6 & 9 & 18 & 19 \\
\hline Adultos & 8 & 7 & 13 & 16 \\
\hline Mayores & 7 & 5 & 6 & 15 \\
\hline
\end{tabular}

\subsection{Procedimiento de BÚSQUedA, CUANTIFICACión Y EXCLUSIONES}

Se realizó la búsqueda de ahora sí que y ahora sí, y sus variantes reducidas fonéticamente ora sí que y ora sí, en los corpora señalados, y las ocurrencias encontradas fueron codificadas por las variables independientes sexo, edad, nivel de instrucción y corpus.

En el proceso de codificación debió decidirse, para cada ocurrencia de ahora sí (que), si se trataba del marcador o de la expresión original con valor temporal y adverbio afirmativo que se compararon en la Sección 3. Se excluyeron de la codificación los casos en los que la construcción era claramente, o podía ser, la construcción original, siguiendo estos criterios: 
a. La existencia de un fragmento previo en el discurso en el que se presenta información igual o muy relacionada con aquella que introduce luego ahora sí (que).

b. En ese fragmento primero se habla del antes, en pasado típicamente, y se contrasta con el ahora.

c. En ese fragmento previo se presenta la información relevante que contrasta, en el plano negación- afirmación (de ahí el sí), con lo que introduce ahora sí (que)

El ejemplo (9) ilustra estos criterios:

(9) I: sí/ porque haz de cuenta que lo grabé

$\mathrm{E}: \mathrm{mh}$

I: para que este/ para poder trabajar/ porque en mi salones// nada más en uno hay para compact < cómpac $>$

$\mathrm{E}: \mathrm{mh}$

I: Entonces lo grabé en un casete/ tres veces/ para que/ los niños trabajaran// y ya/ ese día sí fue así como que/ "bueno/ pues $<\sim$ pus $>$ ahora sí/ pon el disco porque/ se oye más bonito E: sí/ pues sí

I: se oye mejor/ ahí está tu disco/me acuerdas

E: sí/ sí/ no te preocupes (CSCM, Entrevista 46)

En (9) la informante emplea ahora sí para contrastar una situación anterior (antes / ahora), en la que empleaba casetes para oír cierta grabación, con la presente en la que emplea un disco compacto. El contraste que indica el adverbio sí también es accesible: antes no empleaba disco (porque solo en un salón hay aparato reproductor) y ahora sí pone el disco. Ejemplos como (9) son excluidos del conteo porque el significado original de la construcción es muy accesible.

Si no hay un fragmento previo, no se da a. y, por tanto, no se puede dar la relación de b. y c. con una idea previa. En ese caso, ahora sí (que) no tiene su significado original sino que es usado como marcador.

(10) Y eso nomás hasta donde llegamos a saber / eso es malo / si lo llegamos a saber / porque yo / he llegado a saber cosas / que digo / ¿cuánto más / nos faltará saber? / cosas horrendas / y muchas veces los / qu'están más arriba / que tienen... / que / que / que mueren / los gobiernos / son los que / son los que / (...) / ¿por qué? / porque lo pueden hacer / ¿quién los... / quién / quién los... / ora si / como decía una tía mía / ¿quién los 
ataca? / no... / las cosas están / tremendas / ¡ah! / y como les iba diciendo / d'ese discurso que... / que me tocó a mí presentar / entoces ahí / tomando textos de la Biblia / dijo Jesucristo... (CM, Entrevista HM425)

Los casos dudosos surgieron cuando se puede inferir un contenido en el discurso previo que tenga relación temática con el fragmento modificado por ahora sí (que) o se puede inferir, aunque no sea claro, un contraste temporal (ahora / antes) o un contraste afirmación / negación. En una decisión más bien conservadora, los casos dudosos se excluyeron. El ejemplo (11) ilustra este caso.

(11) Inf. A. -No, no, no, no. Auténticas; collares de perlas, de corales, de turquesas, de todo lo que te puedas tú imaginar. $\mathrm{Y}$ el vestido lo tiene esponjado de la cantidad de collares que tiene; porque la gente, cuando le hace algún milagro, en vez de que le cuelguen a ella un milagro ...le cuelgan un collar, eh ...y desde luego, que sí le deben de tener una fe loca, porque debe ser vivísima para haber evitado que su catedral la volaran.

Inf. B. -Cuando hace un milagro, ¡le cuelgan un collar, y uno, cuando hace un milagro...-digo- cuando hace un collar, le cuelgan un milagro.

Inf. A. -Eso sí está pero muy bueno, Güero; ahora sí que estuvo mal tu chiste. Sí, sí, qué curioso ¿verdad? Y que... que... ¡tan increíble la Virgen...este... la Virgen Negra de Barcelona.

Inf. B. -La Moreneta... (CHCM, Norma Culta, Muestra 14)

Es muy dudoso si la expresión en cuestión tiene el valor de marcador en (11) y sí admite, en cambio, la interpretación temporal porque Inf. $A$. contrasta que el intento de chiste por parte de Inf. $B$. fue malo en comparación con intentos pasados, por lo que, en una decisión más bien conservadora, se decide no incluirlo en el conteo de ahora sí (que) como marcador.

Evitaremos a lo largo del análisis hacer uso del concepto de variable pragmática porque no se analiza la elección de dos variantes de un mismo elemento, sino la aparición de la expresión, en términos generales y no en un contexto discursivo concreto en el que pudiera esperarse su aparición y se pueda contabilizar de forma rigurosa su ausencia. Esto hace que, de los tres métodos cuantitativos posibles que Pichler (2010: 591 y sgs.) señala para dar cuenta de las correlaciones entre factores lingüísticos y/o sociales y la variable discursiva, seguiremos la "cuantificación de variables sin un conjunto cerrado de variantes", haciendo uso de frecuencias relativas normalizadas que compensen las diferencias en la cantidad de habla por hablante. 
El cálculo de la frecuencia relativa de ahora sí (que) en los datos, medida normalizada que permitirá comparar los resultados de los diferentes grupos de hablantes (Pichler 2010: 593; Orozco 2014, se calculó en relación con el número de páginas de transcripción en cada entrevista (Orozco 2014) el total de ocurrencias producidas se dividió entre el número de páginas de la entrevista, tomando solo las entrevistas en las que sí se produce ahora sí (que). Para tener números más manejables que los decimales, se multiplicó esa cantidad por mil. La funcionalidad de esta operación radica en el hecho de que permite averiguar si el número de ocurrencias puede estar relacionado con la cantidad de habla producida, de manera que, por ejemplo, si un hablante $x$ produjo 10 ocurrencias de un elemento lingüístico $z$, mientras que $y$ produjo 5 pero $x$ produjo 20 páginas de transcripción e $y 5$, tenemos que el promedio de ocurrencias por página de $x$ es de $0.5 \mathrm{y}$ el de $y$ es de 1. Multiplicando esas cantidades por 1000, obtenemos que el promedio normalizado de ocurrencias producidas por $x$ es de 500 y el de $y$ es de 1000 , es decir, $y$ usa más $z$ que $x$, contrariamente a lo que podría concluirse sin un promedio.

\section{RESULTADOS: DIFERENCIAS DIATÓPICAS Y DIACRÓNICAS EN LA CIUDAD DE MÉXICO Y MONTERREY}

\subsection{MÉXICO}

El conteo de ocurrencias de ahora sí (que) marcador en los corpora de la Ciudad de México arroja la siguiente información, interesante desde el punto de vista diacrónico (Tabla 4).

Tabla 4. Ocurrencias totales de ahora sí (que) en el CSCM y el CHCM

\begin{tabular}{|l|l|l|}
\hline & CSCM & CHCM \\
\hline Ocurrencias & $299(88.72 \%)$ & $38(11.27 \%)$ \\
\hline
\end{tabular}

\footnotetext{
3 Otro cálculo frecuente para obtener una medida normalizada es la proporción por número de palabras. Para una reflexión en torno a este cálculo y sus posibles problemas en inglés, véase Pichler (2010: 593 y sgs.).
} 
El total de ocurrencias del marcador ahora sí (que) encontrado en la Ciudad de México es de 337. Una primera observación en relación con este número, pertinente en tanto que la disciplina discute en este momento las características y bases del estudio de la variación pragmático-discursiva, es que el número total de ocurrencias es bajo, relativamente hablando, si tomamos en cuenta el número de entrevistas consultadas (198 en total) así como su duración (en promedio, aproximadamente 45 minutos). De las 337 ocurrencias totales solo 38 pertenecen al CHCM (recordamos, corpus de los años sesenta y setenta) mientras que el resto, es decir, 299, fueron producidas en el habla recogida en el CSCM. Estos datos muestran una interesante diferencia diacrónica, que se hace aún más visible si tomamos en cuenta la información de la Tabla 5:

Tabla 5. Número de hablantes que emplearon ahora sí (que) en el CSCM y el CHCM

\begin{tabular}{|c|c|c|}
\hline & CSCM & CHCM \\
\hline $\begin{array}{c}\mathbf{N}^{\mathbf{0}} \text { de } \\
\text { Hablantes } \\
(\mathbf{N}=\mathbf{2 3 7})\end{array}$ & $54(108)$ & $17(129)$ \\
\hline
\end{tabular}

La Tabla 5 aborda la cuestión de cuántos hablantes del corpus emplearon el marcador, lo que da otra indicación de su extensión en el habla de la comunidad lingüística estudiada. El número total de hablantes que emplearon el marcador es relativamente bajo en comparación con el número total de hablantes de los dos corpora en conjunto: la mitad de los informantes del CSCM lo emplean, mientras que solo el $13.2 \%$ de los hablantes del corpus del siglo anterior lo hacen. Tenemos, pues, que no solo el número de ocurrencias es más alto en el CSCM que en el CHCM, sino también el número de usuarios que emplea dicho marcador es mayor en el corpus más reciente. Ambas medidas apuntan a una extensión, desde el punto de vista diacrónico, en el empleo de ahora sí (que) en la Ciudad de México.

Una observación pertinente es que, al igual que la frecuencia de los elementos abordados en la variación sociopragmática es más baja que la de los elementos morfosintácticos y, evidentemente, que los fonéticos, también su aparición está más restringida por hablantes. Esto está aún por estudiar en detalle pero probablemente sea un rasgo característico de la variación de marcadores discursivos el que ciertos hablantes no los empleen, o apenas los empleen, mientras que otros los empleen con (mucha) frecuencia. 


\subsection{MONTERREY}

Observemos los mismos datos, número total de ocurrencias por corpus y porcentaje de hablantes que lo emplean, para el uso de ahora sí (que) en Monterrey. La Tabla 6 presenta el número de ocurrencias del marcador en los dos corpora de la ciudad de Monterrey consultados (CM-PRESEEA Y CHM):

Tabla 6. Ocurrencias totales de ahora sí (que) en el CM y el CHM

\begin{tabular}{|c|c|c|}
\hline & $\begin{array}{c}\text { Corpus de } \\
\text { Monterrey - } \\
\text { PRESEEA }\end{array}$ & $\begin{array}{c}\text { Corpus del } \\
\text { Habla de } \\
\text { Monterrey }\end{array}$ \\
\hline Ocurrencias & $28(75.6 \%)$ & $9(24.3 \%)$ \\
\hline
\end{tabular}

Es evidente que en esta ciudad del norte de México el uso del marcador es bastante restringido, pues el número de ocurrencias es sumamente bajo si consideramos el total de entrevistas sociolingüísticas consultadas (262 en total). La comparación entre el número de ocurrencias en los datos de Monterrey y en los de la Ciudad de México es, como se ve, bastante significativa y apunta una clara diferencia diatópica en el empleo de este marcador, al menos en términos de frecuencia.

Respecto a la comparación entre corpora, que nos dará información diacrónica, en el CM-PRESEEA se registraron el triple de ocurrencias de las encontradas en el cHM. Una vez más, la diferencia es bastante reveladora, máxime cuando fueron menos las entrevistas sociolingüísticas del primer corpus (108) que las del segundo (154). Basándonos en estos datos, y a pesar del número tan reducido de ocurrencias, cabe decir que la brecha existente entre el corpus elaborado en los 80 y el contemporáneo es lo suficientemente relevante como para adelantar que el marcador ahora sí (que) está en proceso de expandirse también en Monterrey, si bien su uso es todavía muy restringido. El uso poco frecuente del marcador también se observa en el bajo número de hablantes que lo emplean, como se muestra en la Tabla 7:

Tabla 7. Número total de hablantes que emplearon ahora sí (que) en el CM y el CHM

\begin{tabular}{|c|c|c|}
\hline & $\begin{array}{c}\text { Corpus de } \\
\text { Monterrey - } \\
\text { PRESEEA }\end{array}$ & $\begin{array}{c}\text { Corpus del Habla } \\
\text { de Monterrey }\end{array}$ \\
\hline $\begin{array}{c}\mathbf{N}^{\mathbf{0}} \text { de } \\
\text { Hablantes }\end{array}$ & $13 / 108(12.03 \%)$ & $7 / 154(4.5 \%)$ \\
\hline
\end{tabular}


En el CM-PRESEEA solo el 12.03\% de los entrevistados empleó el marcador y, por su parte, únicamente 7 informantes de 154 lo hicieron en el cHM (apenas el $4.5 \%$ ). Este dato apunta también en la dirección de que, en la Ciudad de Monterrey, el uso del marcador ahora sí (que) es bastante restringido, aunque parece haber experimentado cierta expansión en los últimos años.

\subsection{COMPARACIÓN ENTRE CORPORA}

Para que el análisis de la frecuencia de ahora sí (que) resulte comparable en diferentes corpora, y también para medir el efecto de las variables sociodemográficas en los siguientes apartados, se calculó la medida normalizada como se detalla en el apartado de Metodología.

El número total de ocurrencias apuntaba, en el apartado anterior, a una clara diferencia diatópica en cuanto a frecuencias de ahora sí (que) entre ciudad de México y Monterrey, así como a una diferencia diacrónica en el sentido de un uso más frecuente en los corpora más recientes que en los anteriores. Las Tablas 8 y 9, que incorporan el cálculo descrito, confirman estas diferencias y muestran que la mayor presencia de ahora sí (que) en los corpora más recientes, frente a los anteriores en cada ciudad, no es producto de una diferencia en la longitud de las entrevistas, igual que la mayor frecuencia del marcador en la Ciudad de México frente a Monterrey.

Tabla 8. Promedio ponderado en los corpora de la Ciudad de México

\begin{tabular}{|c|c|c|c|}
\hline \multicolumn{4}{|c|}{ Corpora de Ciudad de México } \\
\hline & CHCM & CSCM & Total \\
\hline $\begin{array}{c}\text { Promedio } \\
\text { normalizado }\end{array}$ & 1998 & 10313 & 12311 \\
\hline
\end{tabular}

Tabla 9. Promedio ponderado los corpora Monterrey

\begin{tabular}{|c|c|c|c|}
\hline \multicolumn{4}{|c|}{ Corpora de Monterrey } \\
\hline & CHM & CM-PRESEEA & Total \\
\hline $\begin{array}{c}\text { Promedio } \\
\text { normalizado }\end{array}$ & 403 & 973 & 1376 \\
\hline
\end{tabular}




\section{LA DISTRIBUCIÓN DE AHORA SÍ (QUE) POR VARIABLES SOCIO-DEMOGRÁFICAS}

\subsection{MÉXICO}

A pesar de que en los datos del Habla Culta y Habla Popular de México las ocurrencias de ahora sí (que) son muy limitadas, ofrecemos aquí la distribución del reducido número de ocurrencias por factores sociodemográficos.

Respecto al nivel de instrucción, hay que recordar que el снсм se compone solo de dos estratos: instrucción alta (Norma Culta) e instrucción baja (Habla Popular). La gran mayoría de las ocurrencias registradas en el CHCM se concentraron en el habla popular $(\mathrm{N}=34,89.4 \%)$ y un mínimo número en la norma lingüística culta. $(\mathrm{N}=4 ; 10.5 \%)$. Hay que recordar que el número de informantes entrevistados en la Norma Culta fue de 87, mientras que en el Habla popular solo fueron 42, de manera que dicha diferencia en el uso se hace más significativa aún ${ }^{4}$. En cuanto a la edad, la distribución de ocurrencias muestra un patrón contrario a lo que haría esperar la diferencia diacrónica entre este corpus y el CSCM: con 20 ocurrencias, los informantes mayores son los que más produjeron el marcador, seguidos de los adultos con 10 y éstos seguidos muy de cerca, a su vez, por lo jóvenes con 8 ocurrencias. El promedio normalizado de ahora sí (que) por longitud de la entrevista confirma esta distribución, en la que los mayores muestran mayor uso de ahora sí (que) (jóvenes, promedio de 367; adultos, promedio de 491; mayores, promedio de 1140). El número tan reducido de ocurrencias en el corpus, sin embargo, no permite obtener claras conclusiones de la distribución social del marcador. Por último, los datos relativos al sexo de los hablantes ofrecen una distribución muy pareja entre hombres (18 ocurrencias) y mujeres (20 ocurrencias).

Más interesante, por el número de ocurrencias encontrado, es el análisis de ahora sí (que) en relación con los rasgos sociodemográficos de los hablantes en el Corpus Sociolingüístico de la Ciudad de México. La distribución de las ocurrencias de ahora sí (que) por nivel de instrucción, edad y sexo se muestran en las Tablas 10, 11 y 12, que incluyen también en la última línea la medida normalizada para cada grupo, calculada como se indicó en la Sección 4.2.

\footnotetext{
4 Solo hay en el CHCM cinco informantes que tienen estudios equivalentes a lo que aquí consideramos nivel medio y ninguno de ellos empleó el marcador.
} 
Tabla 10. Distribución de ahora sí que por nivel de instrucción en CSCM

\begin{tabular}{|l|c|c|c|}
\hline & Nivel alto & Nivel medio & Nivel bajo \\
\hline & $49(16.3 \%)$ & $134(44.8 \%)$ & $116(38.9 \%)$ \\
\hline Promedio & 1801 & 5109 & 3403 \\
\hline
\end{tabular}

Tabla 11. Distribución de ahora sí que por edad en CSCM

\begin{tabular}{|l|c|c|c|}
\hline & Jóvenes & Adultos & Mayores \\
\hline & $175(58.5 \%)$ & $85(28.4 \%)$ & $39(13 \%)$ \\
\hline Promedio & 5976 & 3102 & 1235 \\
\hline
\end{tabular}

Tabla 12. Distribución de ahora sí que por sexo en CSCM

\begin{tabular}{|c|c|c|}
\hline & Hombres & Mujeres \\
\hline & $195(65 \%)$ & $104(34 \%)$ \\
\hline Promedio & 6861 & 3452 \\
\hline
\end{tabular}

La Tabla 10 muestra que las cohortes de instrucción media y baja emplearon el marcador el triple y poco más del doble de veces, respectivamente, en comparación con el grupo con mayor instrucción. En cuanto a la edad, los datos de la Tabla 11 muestran un interesante patrón de cambio aparente en el que la frecuencia de las ocurrencias de ahora si (que) va decreciendo con el aumento de edad: los jóvenes, con diferencia, lo emplean con más frecuencia, después los adultos y, por último, los mayores son el grupo de edad que menos ahora sí (que) emplea. Por último, la distribución por sexo arroja, basándonos de momento en las frecuencias generales (Tabla 12), un mayor uso por parte de los hombres que de las mujeres. Los promedios ponderados confirman exactamente estas distribuciones y descartan que sean debidas a la diferencia de longitudes de las entrevistas de los hablantes.

Resulta interesante observar si estas diferencias por sexo se confirman en todos los niveles de instrucción. El Gráfico 1 muestra el promedio ponderado de ASQ por sexo y nivel de instrucción. 


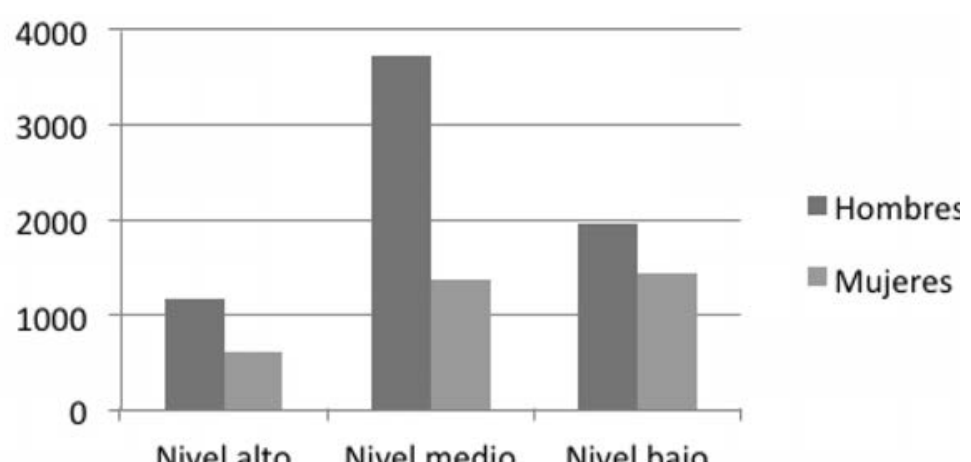

Gráfico 1. Promedio ponderado de acuerdo al sexo y nivel de instrucción

Efectivamente, son los hombres quienes emplean más el marcador en todos los niveles de instrucción: en el nivel alto hay 33 ocurrencias en hombres y 16 en mujeres; en el nivel medio hay 96 en hombres y 38 en mujeres; en el nivel de instrucción bajo, 66 en hombres y 50 en mujeres. El gráfico, empleando el promedio, hace visible también que los hablantes de sexo masculino emplean más ahora sí (que) que las mujeres en todos los niveles. Con el promedio se aprecia, además, que esta diferencia es especialmente relevante para el nivel de instrucción medio y que la diferencia de frecuencias de uso de ahora sí (que) por niveles de instrucción es menor en las mujeres que en los hombres: en ellas la diferencia entre el nivel medio y bajo es prácticamente inexistente, mientras que esta es muy llamativa en los hombres.

Respecto al cruce de las variables nivel de instrucción y edad, se apuntaba en las frecuencias totales un patrón de distribución en el que los jóvenes favorecían ahora sí (que), cuya frecuencia disminuía en los siguientes grupos de edad. La Tabla 13 ofrece el número total de ocurrencias y porcentajes por edad y nivel de instrucción y el Gráfico 2 ofrece los promedios ponderados por estos factores.

Tabla 13. Porcentaje de ocurrencias según la edad y nivel de instrucción

\begin{tabular}{|c|c|c|c|}
\hline & Jóvenes & Adultos & Mayores \\
\hline Nivel alto & $27(55.1 \%)$ & $20(40.8 \%)$ & $2(4 \%)$ \\
\hline Nivel medio & $70(52.2 \%)$ & $43(32 \%)$ & $21(15.6 \%)$ \\
\hline Nivel bajo & $78(67.2 \%)$ & $22(18.9 \%)$ & $16(13.7 \%)$ \\
\hline
\end{tabular}




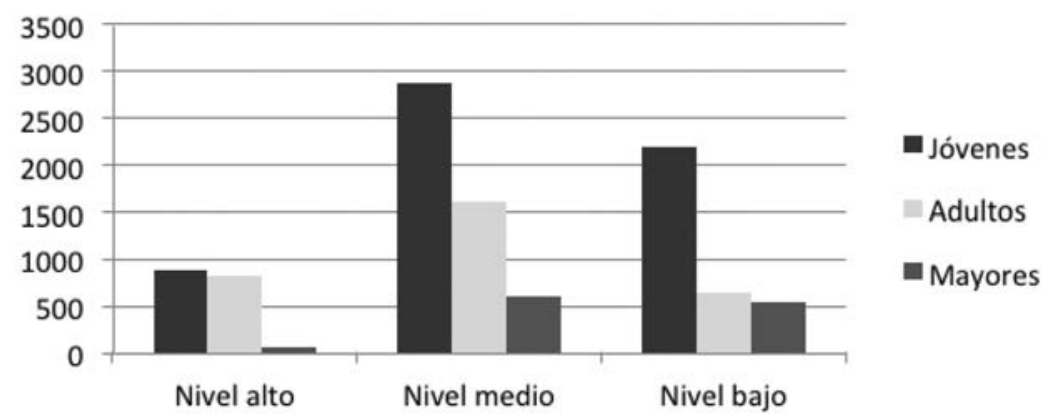

Gráfico 2. Promedio ponderado de acuerdo al nivel de estudios y a la edad

El patrón que las frecuencias totales apuntaban respecto a la edad se confirma para todos los niveles de instrucción. La información del Gráfico 2, además, permite ver que la diferencia entre los jóvenes y el grupo de adultos es mínima en el nivel alto, mientras que es muy significativa en los niveles medio y bajo, especialmente en el primero. Por otra parte, se observa también que la alta frecuencia de ahora sí (que) en hablantes jóvenes se debe, sobre todo, a los hablantes de instrucción media y, en menor medida, a los de nivel bajo de instrucción.

Para concluir el análisis de los datos del Corpus Sociolingüístico de la Ciudad de México, resulta interesante mostrar qué número de hablantes emplearon el marcador de acuerdo al nivel de instrucción y el sexo, para descartar que la utilización del mismo por parte de un pequeño puñado de informantes nos conduzca a concluir afirmaciones poco representativas. $\mathrm{Si}$ recordamos que en este corpus el número de entrevistados es de 6 por casilla y que hay tres grupos etarios, tenemos que para cada nivel de instrucción hay 18 hombres y 18 mujeres. La Tabla 14 recoge el número de usuarios de ahora sí (que) según el nivel de instrucción y sexo:

Tabla 14. Número de hablantes que emplearon el marcador en el CSCM de acuerdo al nivel de instrucción y sexo

\begin{tabular}{|c|c|c|c|c|c|c|}
\cline { 2 - 7 } \multicolumn{1}{c|}{} & \multicolumn{2}{c|}{ Nivel alto } & \multicolumn{2}{c|}{ Nivel medio } & \multicolumn{2}{c|}{ Nivel bajo } \\
\hline Sexo & $\mathbf{H}$ & $\mathbf{M}$ & $\mathbf{H}$ & $\mathbf{M}$ & $\mathbf{H}$ & $\mathbf{M}$ \\
\hline Usuarios & $7 / 18$ & $3 / 18$ & $13 / 18$ & $7 / 18$ & $13 / 18$ & $11 / 18$ \\
\hline
\end{tabular}

Los datos de esta tabla, de nuevo, muestran que la mayor frecuencia de ahora sí (que) se corresponde bastante bien con su expansión entre los hablantes, según las muestras del corpus. En el estrato de instrucción alta es donde se emplea menos el marcador en términos de frecuencias y también es 
donde menos hablantes lo emplean. Los hombres de nivel medio y de nivel bajo presentaban el mayor número de ocurrencias y muestran también la mayor expansión entre hablantes. Por último, las mujeres de nivel medio se alineaban en número de ocurrencias con los hombres de nivel alto, igual que en número de hablantes. El número de hombres que emplean el marcador es también más alto que el de mujeres en todos los estratos.

\subsection{Monterrey}

Como ocurría en los datos del Habla Culta y Habla Popular, el reducido número de ocurrencias de ahora sí (que) en los corpora de Monterrey permite obtener pocas conclusiones de su distribución sociodemográfica. Presentamos, no obstante, aquí los resultados de este análisis cuantitativo.

En el corpus Habla de Monterrey solo hay 9 ocurrencias, cuya distribución es: 4 de hombres y 5 de mujeres. Las 4 de hombres pertenecen a hablantes de instrucción alta, mientras que de las ocurrencias producidas por mujeres, una es en el nivel alto y 4 en el nivel bajo. Respecto a la distribución por edad, 5 son producidas por jóvenes ( 3 en nivel alto, 2 en nivel bajo), tres por hablantes adultos (dos en nivel alto, una en nivel bajo) y una por un hablante mayor de nivel bajo.

En lo que respecta al corpus de Monterrey PRESEEA, se contabilizaron 28 ocurrencias. La Tabla 15 muestra su distribución por edad, nivel de instrucción y sexo del hablante:

Tabla 15. Distribución de las ocurrencias por hablantes según educación, edad y sexo en el CHM

\begin{tabular}{|c|c|c|c|c|c|c|c|c|c|c|c|c|c|c|c|c|c|c|c|}
\hline $\begin{array}{c}\text { Nivel de } \\
\text { instrucción }\end{array}$ & \multicolumn{4}{|c|}{ Nivel alto } & \multicolumn{4}{c|}{ Nivel medio } & \multicolumn{5}{c|}{ Nivel bajo } \\
\hline Edad & Jóv. & Adul. & May. & Jóv. & Adul. & May. & Jóv. & Adul. & May. \\
\hline Sexo & H & M & H & M & H & M & H & M & H & M & H & M & H & M & H & M & H & M \\
\hline Usuarios & 3 & 2 & 2 & 1 & 1 & 0 & 1 & 0 & 0 & 0 & 0 & 1 & 0 & 0 & 1 & 0 & 0 & 1 \\
\hline Ocurrencias & 10 & \multicolumn{2}{|c|}{} & 8 & 1 & \multicolumn{2}{|c|}{6} & 0 & 0 & 1 & & & 1 & 1 \\
\hline
\end{tabular}

Las ocurrencias totales apuntarían a que ahora sí (que) es más frecuente en el nivel alto (especialmente en hombres) que en los demás niveles. El cálculo del promedio ponderado, no obstante, modifica un poco esta figura. 


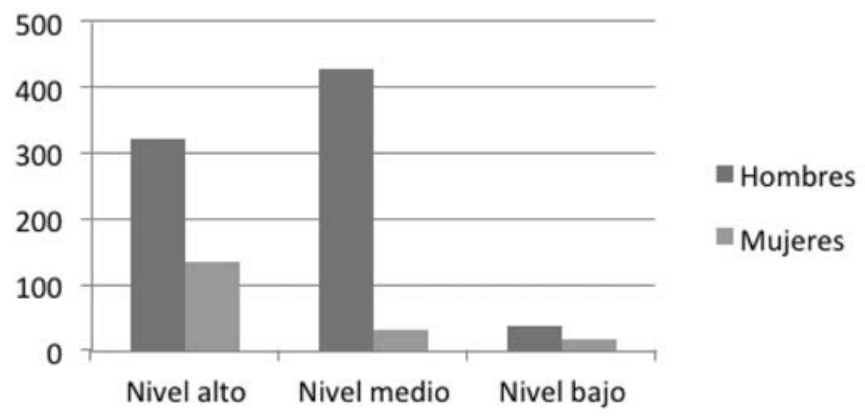

Gráfico 3. Promedio ponderado según nivel de instrucción y sexo

El uso de ahora sí (que) es más alto en las mujeres de nivel alto que en las de medio y bajo, pero en los hombres es en el nivel alto y aún más en el nivel medio, donde la diferencia por sexos es muy acentuada.

Respecto a la edad, las frecuencias totales indicaban que dentro del nivel alto, es el grupo de los jóvenes el que más uso hace de ahora sí (que), dentro del número limitado de ocurrencias. El promedio ponderado indica que jóvenes y adultos tienen un empleo bastante cercano de ahora sí (que) en el nivel de instrucción alto (promedio de cerca de 250 y cerca de 200 respectivamente), mientras que en el nivel de instrucción media, el promedio en los jóvenes se dispara debido a que solo un hablante realiza los seis empleos del marcador. Los datos relativos al número de hablantes que hacen uso de ahora sí (que)(Tabla 15) arrojan también luz sobre la mayor expansión del marcador en el nivel alto en cuanto a su aparición en el habla de más hablantes: un tercio de los hombres entrevistados hace uso del marcador en este nivel, y tres de 18 entre las mujeres, frente a un solo hablante en los demás grupos.

\section{DISCUSIÓN}

Del análisis contrastivo del marcador ahora sí (que) en los dos corpus de PRESEEA analizados, Corpus Sociolingüístico de la Ciudad de México y Corpus de Monterrey, y los dos corpus anteriores de entrevistas sociolingüísticas con los que estos se han comparado, se obtiene información relevante sobre el empleo de este marcador. 
En primer lugar, el análisis de los materiales recogidos en los dos corpus de PRESEEA analizados permite diferenciar el empleo de ahora sí (que) como marcador discursivo frente a su construcción de origen, no gramaticalizada como marcador, aun cuando el análisis de la función o valores de este último no ha sido incluida en este trabajo. Si bien es cierto que las entrevistas sociolingüísticas son un tipo de interacción con características propias, que las diferencian de otras interacciones como la conversación espontánea (Albelda 2004), y esto limita su utilidad para el estudio de muchos fenómenos pragmático-discursivos (por ejemplo, determinados actos de habla, etc.), no debe dejar de valorarse que estos corpora ofrecen datos relevantes para el estudio de marcadores discursivos y otras expresiones con valor pragmático. Es posible, desde luego, que su frecuencia de uso e incluso sus funciones puedan variar entre la entrevista sociolingüística y otras formas de interacción, especialmente si aceptamos, como algunos autores ya proponen (Pichler 2010: 584), que puede ser la diferencia de evento comunicativo lo más relevante en el empleo de marcadores discursivos -frente a los datos macro-sociales-. Esto último, no obstante, es por el momento solo una hipótesis que hay que comprobar comparando corpora que recojan diferentes modalidades de habla y, para esta labor de comparación, la existencia de datos como los recogidos en el Proyecto PRESEEA será, a su vez, de enorme utilidad.

En relación con lo anterior, y dado que existe la lógica impresión de que los marcadores discursivos pueden variar en su uso según el tipo de evento comunicativo que se analice, realizar un estudio contrastivo de variedades de la misma lengua exige contar con corpora que sean comparables en su metodología de recogida. El proyecto PRESEEA ofrece, en este sentido, un invaluable cuerpo de datos que cumple, precisamente, este requisito de comparabilidad, pues son equiparables en todos los parámetros que Pichler (2010: 584) señala: tipo de discurso y contexto de la actividad (comunicativa); tópico, propósito y actitudes en la interacción; roles y relación entre los hablantes y, finalmente, canal comunicativo.

El análisis presentado en el apartado anterior nos ofrece, además de la constatación de la existencia del marcador ahora sí (que), datos relevantes respecto a su variación en el español de México. En primer lugar, la comparación entre los datos recientes, de PRESEEA, y los corpora más antiguos, de los años setenta y ochenta, muestra diferencias de frecuencia de uso significativas: tanto en la Ciudad de México como en Monterrey, aunque con diferentes cifras, el empleo del marcador es más frecuente en los datos más recientes. El marcador discursivo ahora sí (que) parece haberse extendido en su uso en ambas variedades y especialmente en la Ciudad de México. Esta expansión del marcador no se registra solo en una mayor 
frecuencia por número de palabras en las entrevistas, sino en el hecho de que en una mayor proporción de las entrevistas aparece el marcador. Los corpora existentes ofrecen, por tanto, información relevante desde el punto de vista diacrónico.

En segundo lugar, nos resultaba de gran interés comparar el empleo de ahora sí (que) en los dos corpus de PRESEEA en México analizados en este trabajo: la comparación normalizada de la frecuencia de ahora sí (que) muestra una interesante diferencia geográfica en el empleo del marcador, que es mucho más frecuente, según estos datos, en el habla de la Ciudad de México que en la de Monterrey. Esta diferencia se da tanto en los datos más antiguos como en los recientes, pero es en estos últimos en los que resulta especialmente relevante. La interpretación de que el frecuente uso del marcador en el habla de la Ciudad de México esté influyendo en su expansión en Monterrey es tentadora, especialmente porque parecen ser los jóvenes y adultos más educados los que con más frecuencia emplean este marcador en Monterrey, hablantes que podrían tener más contacto con la variedad de la capital del país. Los datos son limitados, pero sí llevan a plantear la pregunta de si estas diferencias de frecuencia y esa distribución en los datos de la variedad con menos ocurrencia de este marcador (¿y otros?) se repetirá entre la Ciudad de México y otras ciudades del país. Los datos que otros equipos de PRESEEA recojan contribuirán a despejar esta duda.

El análisis de ahora sí (que) y los datos sociodemográficos de los hablantes del corpus de la Ciudad de México dan muestra de una distribución por edad en la que el grupo de hablantes jóvenes favorece el empleo de ahora si (que) y el grupo de hablantes mayores es el que menos ahora si (que) utiliza. Este patrón se repite en todos los niveles de instrucción y es en el nivel de instrucción baja el que muestra mayor diferencia entre el grupo joven y los dos siguientes grupos de edad. En una primera interpretación, la mayor frecuencia de ahora sí (que) en los jóvenes es consistente con la diferencia diacrónica que arrojaba el contraste entre ese corpus y el Habla Culta y Habla Popular, indicando un fenómeno nuevo y favorecido por los hablantes de menor edad. La distribución por edad, no obstante, podría indicar tanto un cambio como diferencias generacionales estables.

Teniendo en cuenta el nivel de instrucción de los hablantes, los datos analizados arrojan un patrón en el que es el nivel alto de instrucción el que, con diferencia, menor uso hace de ahora sí (que), mientras que el de instrucción media es el que más lo emplea, seguido de cerca por el nivel bajo. Los datos del nivel medio de instrucción parecen deberse, sobre todo, al uso muy frecuente de ahora sí (que) por los hombres de este estrato educativo. Si se trata de un cambio, este se está produciendo desde las clases menos instruidas. La diferencia entre hombres y mujeres añade información útil 
para la interpretación de los resultados: en los tres niveles de instrucción, las mujeres emplean menores porcentajes de ahora sí (que) que los hombres y hay pocas diferencias en el uso de las mujeres de instrucción baja y las de instrucción media. Este panorama de la estratificación social de ahora sí (que) parece indicar, a la espera de algún estudio que aporte datos directos sobre actitudes hacia este marcador, que ahora si (que) supone una innovación lingüística que no goza de gran prestigio en la comunidad, pues ni los hablantes de instrucción alta, ni las mujeres, que como se sabe suelen ser favorecedoras de las variantes de prestigio en su comunidad, favorecen ahora si (que) (Labov 1990: 210 y sgs, Cheshire 2008).

El análisis presentado en este trabajo permite constatar, además, algunos aspectos problemáticos o algunos retos de la investigación de variación pragmática. En primer lugar, si en los estudios de variación morfosintáctica suponía ya un reto el hecho de que las variables son poco frecuentes, este problema es aún mayor en los estudios que observan la frecuencia de unidades como los marcadores discursivos, como este trabajo ilustra.

En segundo lugar, este trabajo ha abordado el análisis de los factores sociales disponibles en los corpora sin distinguir las distintas funciones que ahora sí (que) pueda estar realizando en el discurso. Cabe la posibilidad, por tanto, de que, si ahora sí (que) muestra tener diversas funciones o valores en el discurso, los datos que los corpus nos ofrecen tuvieran que ser analizados con más detalle para explorar si, por ejemplo, es concretamente una de las funciones la que muestra determinada distribución social. Solo más estudios de variación, empleando más datos, y una descripción detallada del valor de ahora sí (que) permitirán profundizar en esta línea de análisis.

Por último, uno de los aspectos que más discusión teórica merece en los pocos trabajos que hasta el momento se dedican a este tipo de estudios es la conveniencia o posibilidad de expandir el concepto de variable sociolingüística al nivel pragmático-discursivo. Como mencionábamos, la discusión tuvo lugar ya respecto a la variación morfosintáctica, pero en este nivel de análisis lingüístico la expansión del concepto de variable se dibuja, al menos de momento, mucho más problemática. Por un lado, como ocurría en el nivel morfosintáctico frente al fonético pero ahora con mayor complejidad, está el "problema del significado" que, no obstante, no parece ser la dificultad mayor en este caso. Una vez que asumimos, en la variación morfosintáctica, que las variantes de una variable no son realmente, en muchos casos, dos maneras de "decir la misma cosa" sino que, de hecho, estarán con frecuencia asociadas a diferencias de significado pragmáticodiscursivo (Schwenter 2011), y que el análisis cuantitativo es la herramienta necesaria que nos permitirá discernir estas sutiles diferencias de significado, esa misma estrategia es extrapolable -quizá no sin matizaciones- a este 
nivel de análisis: las dos -o más- formas lingüísticas que alternan están, probablemente, asociadas a diferencias pragmáticas y el estudio cuantitativo, incluyendo como variables independientes factores pragmático-discursivos, permitirá acercarnos a estas diferencias que no son capturables basándonos solo en la introspección. Probablemente el mayor problema para expandir el concepto de variable al nivel pragmático-discursivos es, precisamente, determinar la variable y sus variantes: ¿cuándo nos encontramos ante dos o más expresiones que sea pertinente relacionar como variantes de una variable? ¿Cuál es el criterio? La discusión detallada de este asunto está fuera de los límites de este artículo pero, como Pichler $(2010)^{5}$ señala, los trabajos que se están realizando hasta la fecha varían, en la práctica, en el criterio que se toma al respecto y, a su vez, estas diferencias de criterio condicionan, en buena medida, los posibles cálculos cuantitativos que cada estudio permite realizar. En nuestro caso, se analiza la presencia del marcador ahora sí (que) sin hacer uso explícito, y esto de forma intencionada y consciente, del concepto de variable. Se trata, por tanto, de un análisis que encaja en la "cuantificación de variables sin un conjunto cerrado de variantes" en la tipología de Pichler. Somos conscientes de las limitaciones de este tipo de análisis pero lo consideramos un primer paso necesario para aproximarnos a este tipo de estudios. En una etapa posterior, cuando se cuente con más datos de las mismas variedades, será interesante abordar el análisis diferenciando los usos con reducción formal (ora sí) de los usos sin reducción formal (ahora sí que) de este marcador y considerando a ambos como variantes de una misma variable. En este punto de la investigación, no obstante, se descartó esta posibilidad dado el reducido número de ocurrencias de la forma reducida.

\section{CONCLUSIONES}

Este trabajo parte de la visión de que la lengua es inherentemente variable y que la variación se da en todos los niveles de análisis lingüísticos. Las páginas anteriores han presentado un acercamiento a la variación existente en el empleo del marcador discursivo ahora sí (que) en dos variedades del español de México. 
La comparación de corpus de dos ciudades mexicanas -Ciudad de México y Monterrey- recogidos en dos momentos diferentes ha permitido constatar diferencias diacrónicas y diatópicas claras y apuntar interesantes diferencias sociales en el empleo del marcador en la Ciudad de México, donde su empleo está favorecido por hablantes jóvenes, por hombres y por informantes de los niveles de instrucción bajo y medio.

Este análisis ha mostrado la utilidad de los corpus sociolingüísticos para explorar, al menos en un primer acercamiento, el empleo y distribución social de marcadores discursivos y, en concreto, la utilidad que los corpus recogidos en el proyecto PRESEEA, por sus características, ofrecen a la comunidad para abordar, además de la variación fonética y morfosintáctica, la variación de expresiones con valor pragmático. Esta labor, que está aún en una etapa muy preliminar dentro del vasto campo de la investigación sociolingüística y la investigación pragmática, requiere sin duda de más análisis que nos ayuden a dibujar un más preciso panorama de las características que la variación en los marcadores discursivos -en el sentido más amplio- tiene respecto a otros tipos de variación y de la forma en la que el análisis cuantitativo de corpus reales puede enriquecer la descripción de diferencias pragmático-discursivas que difícilmente capturan los estudios cualitativos.

\section{REFERENCIAS BIBLIOGRÁFICAS}

Albelda Marco, Marta. 2004. Cortesía en diferentes situaciones comunicativas. La conversación coloquial y la entrevista sociológica semiformal. En D. Bravo y A. Briz (eds.). Pragmática sociocultural. Estudios sobre cortesía en español, pp. 109-134. Barcelona: Ariel.

Álvarez, Alexandra y Carolina Joven. 2005. Atenuantes y sus funciones corteses. Manipulación y seducción en conversaciones entre mujeres de Mérida. En D. Bravo (ed.). Estudios de la (des)cortesía en español, pp. 119-144. Estocolmo, Buenos Aires: Dunken.

Andersen, Gisle. 2001. Pragmatic markers and sociolinguistic variation. Amsterdam/ Filadelfia: John Benjamins.

Bybee, Joan. 2006. From usage to grammar: The mind's response to repetition. Language 82: 711-733.

Carbonero Cano, Pedro y Juana Santana Marrero. 2010. Marcadores del discurso, variación dialectal y variación social. En O. Loureda Lamas y E. Acín Villa (coords.). Los estudios sobre marcadores del discurso en español, hoy. Madrid: Arco/Libros.

Cepeda, Gladis y María Teresa Poblete. 1999. Los marcadores discursivos en el habla femenina de Valdivia (Chile): nivel léxico y suprasegmental. En Actas del Congreso Interancional de la Asociación de Lingüística y Filología de la América Latina, pp. 591598. Las Palmas de Gran Canaria: Universidad de Las Palmas de Gran Canaria, vol. I.

Cestero Mancera, Ana María y Francisco Moreno Fernández. 2008. Uso y funciones de vale y ¡venga! en el habla de Madrid. Boletín de Lingüística XX: 64-84.

Cheshire, Jenny. 2007. Discourse variation, grammaticalisation and stuff like that. Journal of Sociolinguistics 11: 155-193. 
2008. Sex and gender in variationist research. En J. K. Chambers, P. Trudgill y N. Schilling-Estes. The handbook of language variation and change, pp. 423-443. Oxford: Blackwell.

Cortés Rodríguez, Luis. 1991. Sobre conectores, expletivos y muletillas en el español hablado. Málaga: Ágora.

1998. Marcadores del discurso y análisis cuantitativo. En M. A. Martín Zoraquino y E. Montolío Durán (coords.). Los marcadores del discurso. Teoría y Análisis, pp. 143160. Madrid: Arco / Libros.

Dines, Elizabeth R. 1980. Variation in Discourse: “And Stuff like That”. Language in Society 9:13-31.

Dubois, Sylvie. 1992. Extension particles, etc. Language Variation and Change 4: 179-203.

ERman, Britt. 2001. Pragmatic markers revisited with a focus on you know in adult and adolescent talk. Journal of Pragmatics 32: 1337-1359.

Fuentes, Catalina 1990a. Procedimientos intradiscursivos: decir y los explicativos. En P. Carbonero y M. T. Palet (eds.). Sociolingüística Andaluza 5: habla de Sevilla y hablas americanas, pp. 103-123. Sevilla: Publicaciones de la Universidad.

1990b. Algunos operadores de función fática. En P. Carbonero y M. T. Palet (eds.). Sociolingüistica Andaluza 5: habla de Sevilla y hablas americanas, pp. 137-170. Sevilla: Publicaciones de la Universidad.

1993a. Claro: modalización y conexión. En P. Carbonero y C. Fuentes (eds.). Sociolingüistica Andaluza 8: estudios sobre el enunciado oral, pp. 99-126. Sevilla: Publicaciones de la Universidad.

1993b. Desde luego, por supuesto, naturalmente. En P. Carbonero y C. Fuentes (eds.). Sociolingüistica Andaluza 8: estudios sobre el enunciado oral, pp. 127-159. Sevilla: Publicaciones de la Universidad.

Garcés Gómez, María Pilar. 1994a. Funciones y valores de entonces en el español hablado. En M. Alvar Ezquerra y J. A. Villena Ponsoda (coords.). Estudios para un corpus del español, pp. 217-231. Málaga: Universidad de Málaga.

1994b. Elementos de cohesión en el español hablado: pues. En M. Alvar Ezuerra y J. Andrés Villena Ponsoda (coords.). Estudios para un corpus del español, pp. 231-244. Málaga: Universidad de Málaga.

Labov, William. 1990. The intersection of sex and social class in the course of linguistic change. Language Variation and Change 2, 205-254.

LaVANDERA, Beatriz. 1978. Where does the sociolinguistic variable stop? Language in Society 7: $171-182$.

Lope Blanch, Juan Manuel. 1971. El habla de la ciudad de México. Materiales para su estudio. México: UNAM. Versión en línea disponible en: http://www.iifilologicas.unam. $\mathrm{mx} /$ elhablamexico/index.php?page=norma-culta

1976. El habla popular de la ciudad de México. Materiales para su estudio. México: UNAM. Versión en línea disponible en: http://www.iifilologicas.unam.mx/elhablamexico/ index.php?page $=$ habla-popular

Martín Butragueño, Pedro y Yolanda Lastra (coords.). 2011. Corpus sociolingüístico de la ciudad de México. Vol. I: Nivel alto (México: El Colegio de México, 2011, 103 pp. + 1 CD).

2012. Corpus sociolingüistico de la ciudad de México. Vol. II: Nivel medio (México: El Colegio de México, 2012, 95 pp. + 1 CD).

Corpus sociolingüistico de la ciudad de México. Vol. III: Nivel bajo (México: El Colegio de México, en preparación).

Martín Zorraquino, María Antonia y Estrella Montolío (coords.). 1998. Los marcadores del discurso. Teoría y análisis. Madrid: Arco/Libros.

Martín Zorraquino, María Antonia y José Portolés. 1999. Los marcadores del discurso. En I. Bosque y V. Demonte (dirs.). Gramática descriptiva de la lengua española. Madrid: Espasa Calpe, capítulo 63. 
Murillo Ornat, Silvia. 2000. English explicatory reformulative discourse markers: Conceptual or procedural? En M. P. Navarro, R. Lorés Sanz, S. Murillo Ornat y C. Buesa (eds.). Transcultural communication: Pragmalinguistic Aspects, pp. 131-138. Zaragoza: Anubar.

Orozco, Leonor. 2014. El empleo de ¿no?, ¿eh? y ¿verdad? en situación de entrevista sociolingüística. En P. Martín Butragueño y L. Orozco. (eds.). Argumentos cuantitativos y cualitativos en sociolingüística. México, D.F.: El Colegio de México.

Pichler, Heike. 2010. Methods in Discourse Variation Analysis: Reflections on the Way Forward. Journal of Sociolinguistics 14/5: 581-608.

2013. The Structure of Discourse-Pragmatic Variation. Amsterdam/ Philadelphia: John Benjamins Publishing.

Pichler, Heike y Stephen Levey. 2011. In search of grammaticalization in synchronic dialect data: general extenders in northeast England. English Language and Linguistics 15: 441-471.

Portolés LÁzAro, José. 1998. Marcadores del discurso. Barcelona: Ariel.

PRESEEA. Proyecto sociolingüistico para el español de España y de América. Página web: http://preseea.linguas.net/Inicio.aspx, consultada en junio de 2015.

Rodríguez Alfano, Lidia. 2010. Compilación digital del corpus de El habla de Monterrey, con Registro de Derechos de Autor SEP: 03-2010-070613330700-01

Rodríguez Alfano, Lidia, Flores Treviño, M. E. y Pérez Aguirre, T. (comps.). 2012. Corpus Monterrey-PRESEEA. Monterrey: Facultad de Filosofía y Letras/Universidad Autónoma de Nuevo León.

Rodríguez Cadena, Yolanda. 1999. Marcadores discursivos en el habla de Barranquilla. Estudio sociolingüístico. Lotterae 8: 197-220.

San Martín, Abelardo. 2004-2005. Igual como marcador discursivo en el habla de Santiago de Chile: función pragmático-discursiva y estratificación social de su empleo. Boletín de Filología XL: 201-232.

2013. Los reformuladores de distanciamiento en el corpus PRESEEA de Santiago de Chile. Boletín de Filología XLVIII: 171-199.

Santana Marrero, Juana. 2014. Marcadores del discurso de Sevilla. Cuadernos de la ALFAL 5: $277-311$.

SAntos Río, Luis. 2003. Diccionario de partículas. Salamanca: Luso-Española de Ediciones.

SCHNEIDER, KLAus P. 2010. Variational pragmatics. Variation and Change. En Mirjam Fried, Jan-Ola Östman y Jef Verschueren. Pragmatic perspectives, pp. 239-267. Amsterdam / Filadelfia: John Benjamins.

Schwenter, Scott A. 2011. Variationist Approaches to Spanish Morphosyntax: Internal and External Factors. En Diaz Campos, M. Handbook of Hispanic Sociolinguistics, pp. 123147 Oxford: Blackwell.

Serrano, María José. 1995. El uso de la verdad y pues como marcadores discursivos de respuesta. Español Actual 64: 5-16

2001. The socio-communicative function of two discourse markers in Spanish. Estudios de Sociolingüística 2/1: 101-122.

Silva Corvalán, Carmen. 2001. Sociolingüistica y pragmática del español. Washington, DC: Georgetown University Press.

Tagliamonte, Sali. 2005. So who? Like how? Just what? Discourse Markers in the Conversations of Young Canadians. Journal of Pragmatics 37: 1896-1915.

Terkourafi, Marina. 2009. Variationist methodology in pragmatics: (how) can it be done?, Proceedings from the Annual Meeting of the Chicago Linguistic Society, Volume 45, Number 1, 1 January 2009, pp. 609-623 (15). 